\title{
WEAK SIGNAL DETECTION BASED ON MULTIPLE AUTO-CORRELATION TECHNIQUES
}

\author{
'Sarah S. Mohammed ${ }^{1}$
}

Maher K. Mahmood1

1) Electrical Engineering Department, College of Engineering, Mustansiriyah University, Baghdad, Iraq

\begin{abstract}
This study presents the performance of the auto-correlation methods for detecting weak signals, where the signal level is much less than the noise level. Double and triple auto-correlation techniques are used to improve the detection performance compared with the single autocorrelation. Simulation results obtained by MATLAB programs show that the multiple correlation techniques outperform the single correlation in terms of probability of detection and probability of false alarm versus signal to noise ratio SNR.
\end{abstract}

Keywords: weak signal detection, signal to noise ratio (SNR), auto-correlation method, triple auto-correlation.

\section{Introduction}

Recently, there has been an increase in the studies of acquiring, analyzing and processing all kinds of information in a deeper way, and in particular there is a great interest in discovering useful weak signals embedded in strong noise. A weak signal is a signal that has a signal-to-noise ratio less than zero $\mathrm{dB}$, meaning the noise level is bigger than the signal level. The importance of these researches is evident in its wide applications such as very longdistance communication when the signal power becomes very low like satellite communication, seismic testing, pipeline leakage, fault diagnosis of mechanical system etc. Hence, the detection of the weak signal under low signal-to-noise ratio becomes very import-ant. Accordingly, several methods of processing the weak signal, including neural network, wavelet analysis method and fuzzy set theory [1,2], have been developed.
Le et al. [3] suggested the inner product vector for detection where cross correlation functions between response measurements is used. Where the response quantity is due to white noise excitation. Avramchuk et al. [4] made the analysis of the correlation of two signals, to determine valid signal frequency limits and to set on this basis frequency filters parameters to enhance the signalto-noise ratio. Liu et al. [5] suggested a new method to detect unknown weak signals in the satellite applications. These methods depends on cross-correlation algorithm, which does not need previous knowledge of the signal, only resorts to the features of signal to filter noise and detect if the unknown signal exists or not. On the other hand, some methods of weak signal detection need a certain previous knowledge of the signal, i.e., some information of the signal must be known right from the beginning, such as frequency, phase, etc. Blind detection techniques based on auto-correlation algorithms are given in [6,7] where these techniques do not require prior knowledge of the signal parameters.

Yuandong et al. [8] suggested a detector based on Ensemble Average of Autocorrelated Envelopes to identify the early occurrence faults in rolling element bearings, of which the fault caused vibration signals are contaminated or masked by additive background noise and random phase

*Corresponding Author: sarahsabah949@gmail.com 
noise (or slippage between bearing components). To improve the cyclostationary characteristics, it uses the phase synchronization property of autocorrelation for aligning the cyclostationary signals in the lag domain to obtain an effective ensemble average which permits both types of random effects to be eliminated significantly. Duona et al. [9] suggested the learning modulation filter networks to enhance the detection performance. The approach depends on a two-stage optimization scheme for filter learning, attention mechanism and classification in a unified framework.

The main challenging problem in detection of weak signals is to get high detect probability with low probability of false alarm at low signal to noise ratio.

In this paper, multiple autocorrelation techniques are used to improve the detection performance over the single auto-correlation techniques. MATLAB simulation results assure that the double and triple auto- correlation techniques outperform the single autocorrelation in terms of probability of detection $P_{d}$ and probability of false alarm $P_{f a}$ versus the signal to noise ratio SNR.

The rest of this paper proceeds as follows: Section 2 discusses the theory of autocorrelation; Section 3 discusses the simulation results of the techniques; Section 4 gives some conclusions.

\section{Weak Signal Detection Based on Auto- Correlation Technique}

\subsection{Auto-Correlation Method}

The techniques based on correlation method to detect weak signals depends on the statistical characteristics of the SNR, and the correlation function is a measure of the similarity between two signals [10].
Often the values of deterministic signal have a stronger correlation at different times. On the contrary, the noise values are random and therefore has less correlation. By using these properties (the difference between the signal and the noise), the deterministic signal and random noise can be distinguished. So, we can detect the weak signals from strong noise based on this theory. An autocorrelation function is expressed as [11]:

$R_{x x}(\tau)=\int_{-\infty}^{\infty} f(t) * f(\tau+t) d t$

Where $R_{x x}$ is autocorrelation function, $\tau$ is lag index, $\mathrm{f}(\mathrm{t})$ is received signal, $*$ is complex conjugate of the signal. Autocorrelation method can be achieved in time or frequency domain [12]. Autocorrelation function has another property, which is the symmetry with respect to the origin, maximum at $(\tau=0)$, periodicity in the case of pure signals, and attenuation in the case of AWGN noise as shown in Fig. 1, where:

$$
\begin{aligned}
& R_{x x} \neq 0 \ldots \tau=0(\max ), \\
& R_{x x} \approx 0 \ldots \tau \neq 0
\end{aligned}
$$

Fig. 2 shows autocorrelation of the periodic signal where it has spike form of correlation function at $(\tau=0)$, and other lags values for $(\tau \neq 0)$. These lag values roll away from lag $(\tau=0)$. The magnitudes of these lags depend on the feature of the data which consists of the interested signal.

$$
\begin{aligned}
& R_{x x} \neq 0 \ldots \tau=0(\max ), \\
& R_{x x} \neq 0 \ldots(\tau \text { closet } \text { to } 0)
\end{aligned}
$$

Hence, and as shown in Fig.1, one can use other than the zero lag to detect the presence of the signal. 
(a)

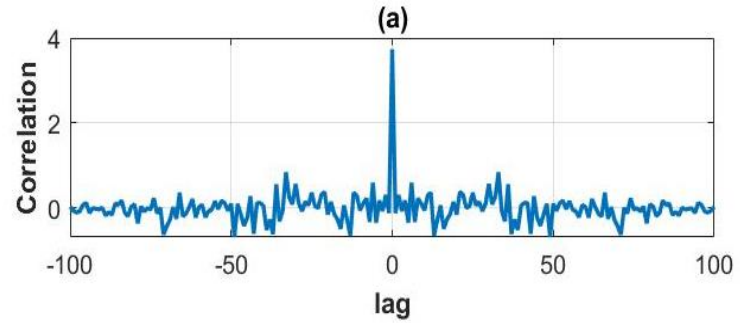

(b)

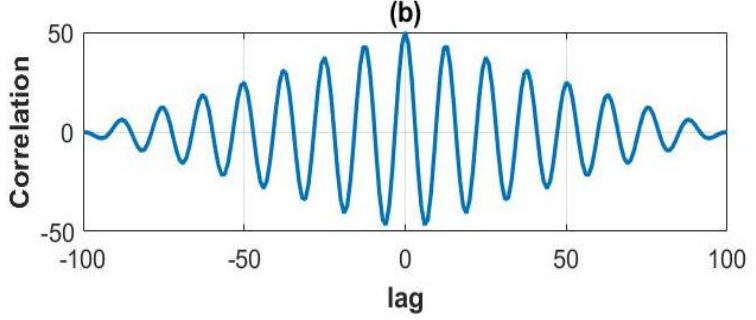

Figure 1. Auto-correlation function of (a) AWGN; (b) signal $f(t)$.

\subsection{Multiple Auto-correlation}

However, and under very low signal to noise ratio, this non-zero lag value may be deeply embedded in noise and may be lost.

The detection performance of the signal $s(t)$ embedded in the noise $n(t)$ can be further improved if double or even triple autocorrelation computation are carried out i.e., the process is shows block diagram for the three levels of autocorrelation.
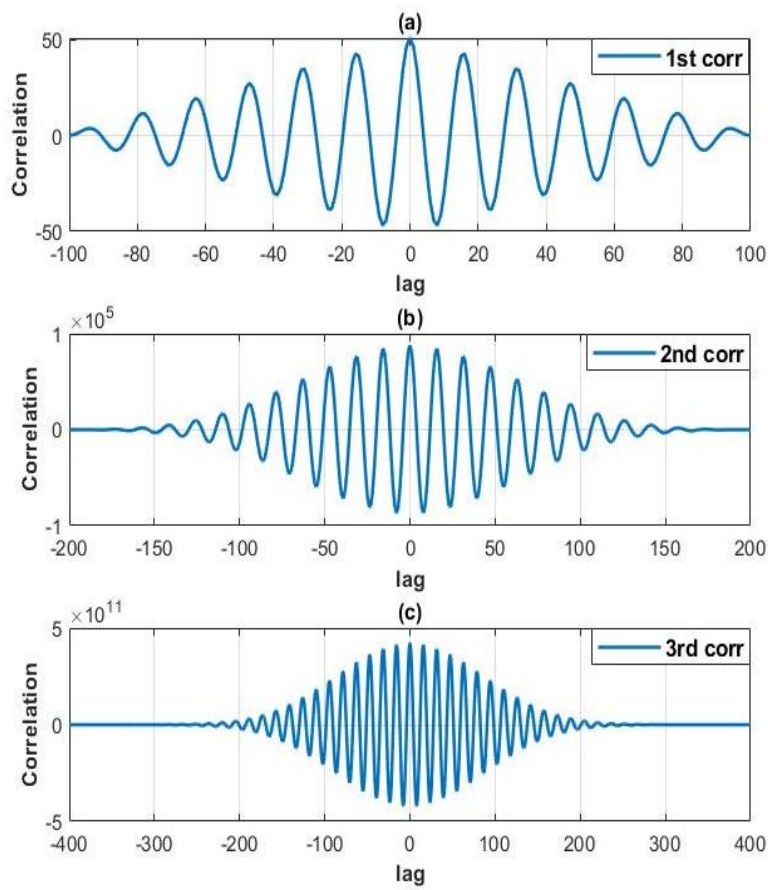

Figure 2. Auto-correlation function of signal (a) single auto-correlation; (b) double auto-correlation; (c) triple auto-correlation.

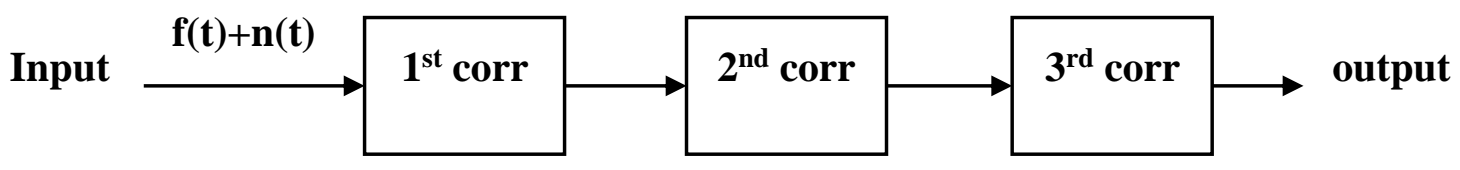

Figure 3. Block diagram for multiple Auto-correlation.

Repeated to obtain an improvement in the signalto-noise ratio and to determine the spectral content of the signal [11]. By using the previous properties of auto-correlation function, it is possible to detect weak signal better than a single and double auto- correlation Where the number of harmonics doubles and the amplitude of the signal increases dramatically when the correlation is repeated as shown in Fig.2. Fig.3

\section{Simulation Results}

According to what was mentioned in the previous paragraph of the characteristics of autocorrelation, where all the harmonics of the noise are almost zero except for lag $(\tau=0)$, and also the auto-correlation function of the periodic signal gives a large harmonic value, but it is smaller as it moves away from lag $(\tau=0)$. Using this feature, the detection process is done by taking a specific 
lag peak (say $\tau=1$ ) and operating the system with noise only, setting a threshold value to obtain the required probability of false alarm $P_{f a}$, and then the signal to be detected is added to the noise.

The system uses the same threshold value set to obtain a certain $P_{f a}$ when the signal is embedded in the noise and the system has to discover the presence of the information signal with a certain $P_{d}$. The de-noising performance of the double and triple auto-correlation is evaluated and compared with single auto-correlation. The tested signal is a sinusoid with amplitude $A=1$ volt and normalized frequency $\mathrm{f}=1 \mathrm{~Hz}$ with the data length $\mathrm{N}=10000$ as shown in Fig. 4(a). An AWGN with $\mathrm{SNR}=-4 \mathrm{~dB}$ is added to the pure signal as illustrated in Fig. 4(b). Fig. 4(c)-(d) display the single and double auto-correlation method respectively, for which the periodical harmonic signal is almost recovered from the noisy signal. By employing the triple autocorrelation method, the periodical harmonic signal is better than the result obtained in single and double auto-correlation method as demonstrated in Fig. 4(e) where the detection performance is largely improved. Fig. 5 is similar to Fig. 4 with $\mathrm{SNR}=-20 \mathrm{~dB}$.

The performance of $P_{d}$ versus SNR under different $P_{f a}$ are given in Fig. 6(a)-(b)-(c). The input SNR varies from -20 to $0 \mathrm{~dB}$, from -23 to $0 \mathrm{~dB}$ and -28 to $0 \mathrm{~dB}$ for single, double and triple auto-correlation respectively. As an example, for $\mathrm{SNR}=-20 \mathrm{~dB}$ with $P_{f a}=0.05$ at single autocorrelation $P_{d}=20 \%$ and at double autocorrelation $P_{d}=100 \%$, and at $\mathrm{SNR}=-25 \mathrm{~dB}$ with the same $P_{f a}$ at double auto-correlation $P_{d}=29 \%$ and at triple auto-correlation $P_{d}=79 \%$.
To further evaluating the detection performance, the receiver operating characteristic (ROC) curves at $\mathrm{SNR}=-13,-15$ and $-17 \mathrm{~dB}$ are presented in Fig. 7(a) for single auto-correlation. Fig. 7(b) presented the ROC curves for double autocorrelation at $\mathrm{SNR}=-21,-22$ and $-23 \mathrm{~dB}$. Fig. 7(c) shows the ROC curves for triple auto-correlation under lower SNR =-24, -25 and $-27 \mathrm{~dB}$.
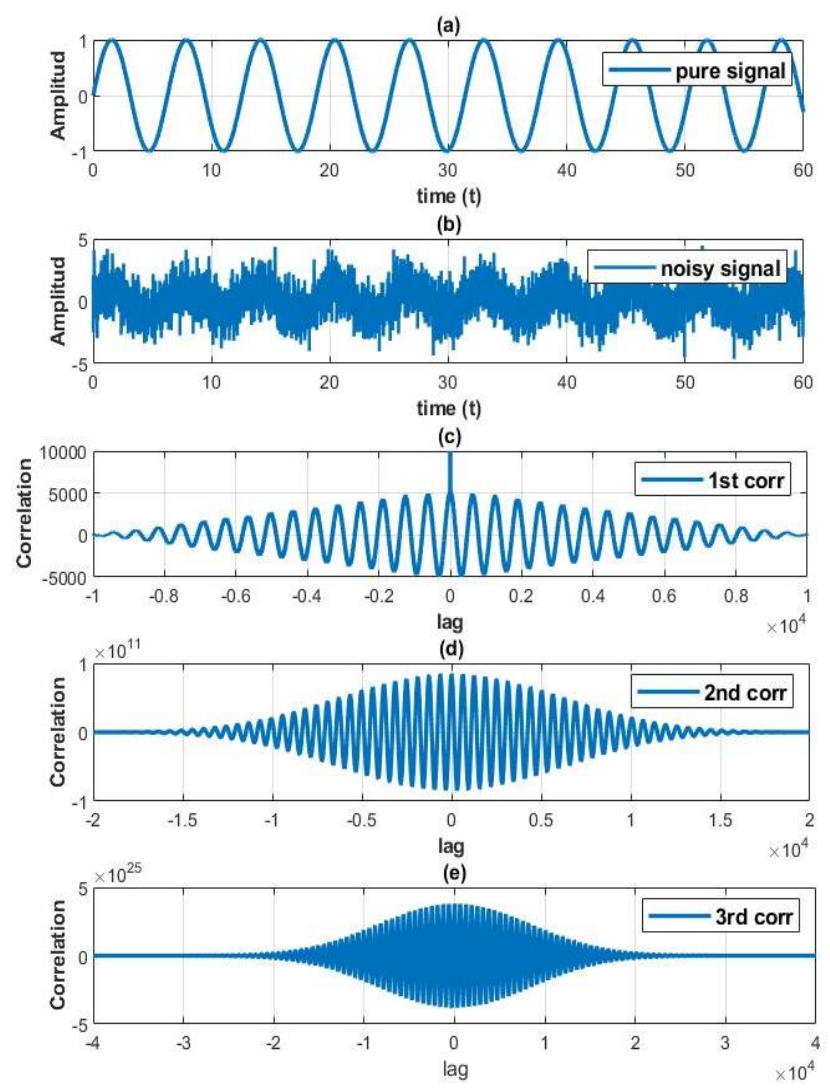

Figure 4. Auto-correlation function of signals with $\mathrm{SNR}=-$ $4 \mathrm{~dB}$ (a) pure signal; (b) noisy signal; (c) Auto-correlation to noisy signal after first stage of correlation (d) Autocorrelation to noisy signal after second stage of correlation. (e) Auto-correlation to noisy signal after third stage of correlation. 


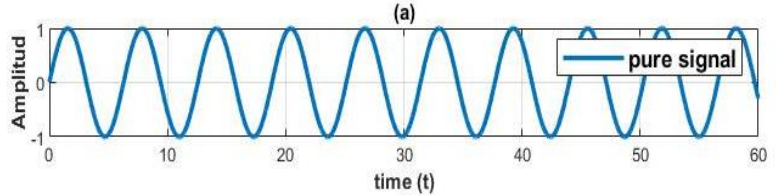

(b)
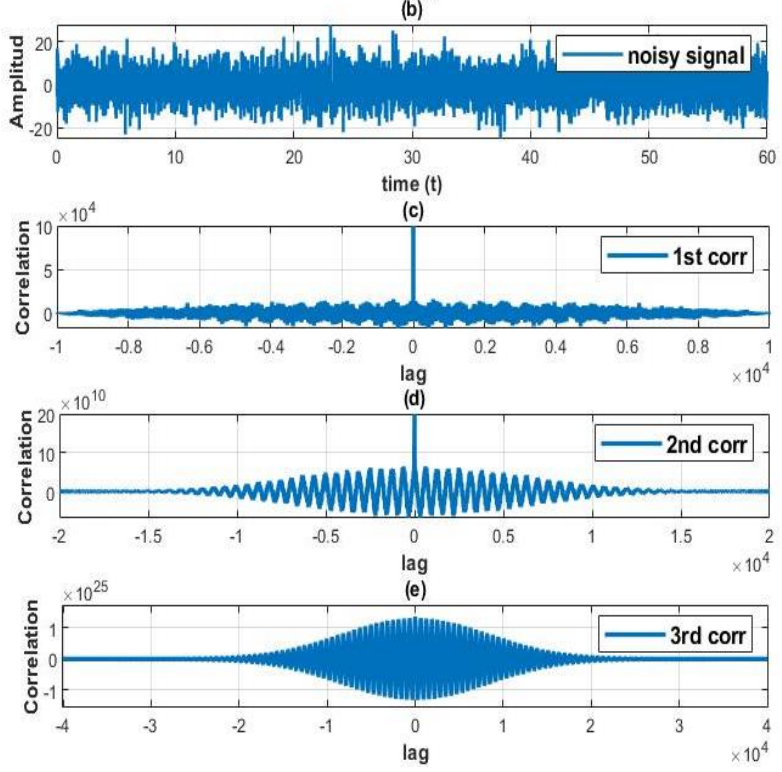

Figure 5. Auto-correlation function of signals with $\mathrm{SNR}=-$ $20 \mathrm{~dB}$ (a) pure signal; (b) noisy signal; (c) Auto-correlation to noisy signal after first stage of correlation (d) Autocorrelation to noisy signal after second stage of correlation. (e) Auto-correlation to noisy signal after third stage of correlation.

Fig. 8 demonstrates the differences between single, double and triple correlation techniques at $P_{f a}=0.05$. One can easily notice that for $P_{d}=0.9$, the double auto-correlation is approximately $6 \mathrm{~dB}$ better than single and triple auto-correction is even better than double by $3 \mathrm{~dB}$. Table (1) shows a comparison of the SNR requirement for $P_{d}=0.9$ for $P_{f a}=0.01,0.05$ and 0.1 .

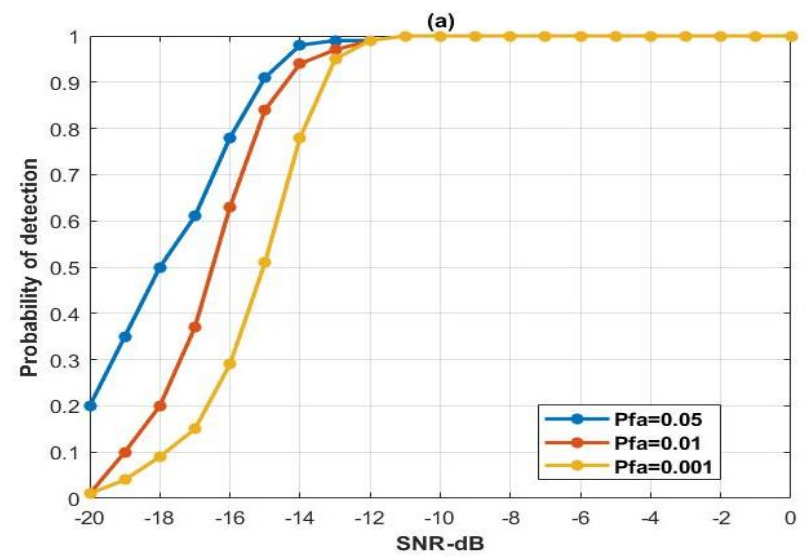

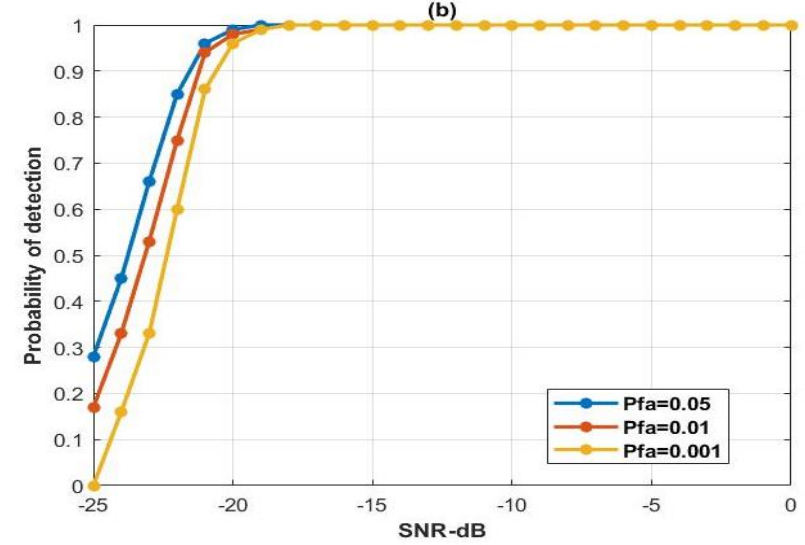

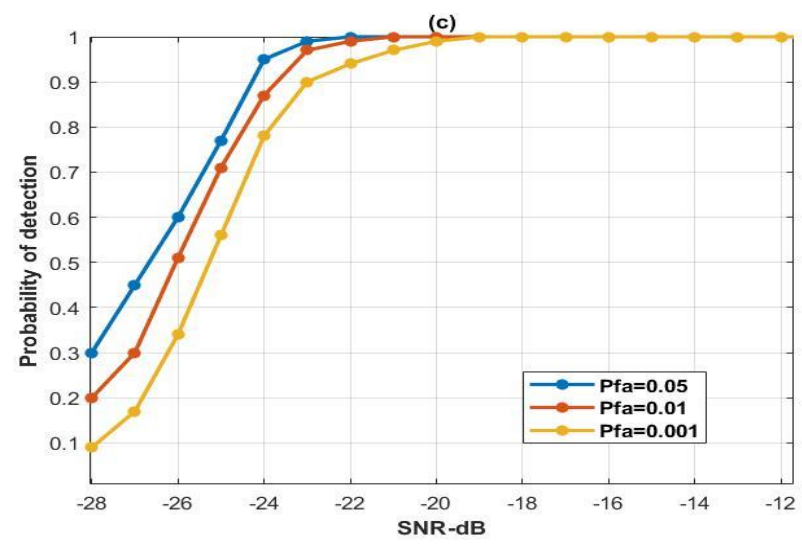

Figure 6. Probability of detection versus SNR for single tone detection at using autocorrelation, (a) single autocorrelation; (b) double auto-correlation; (c) triple autocorrelation.

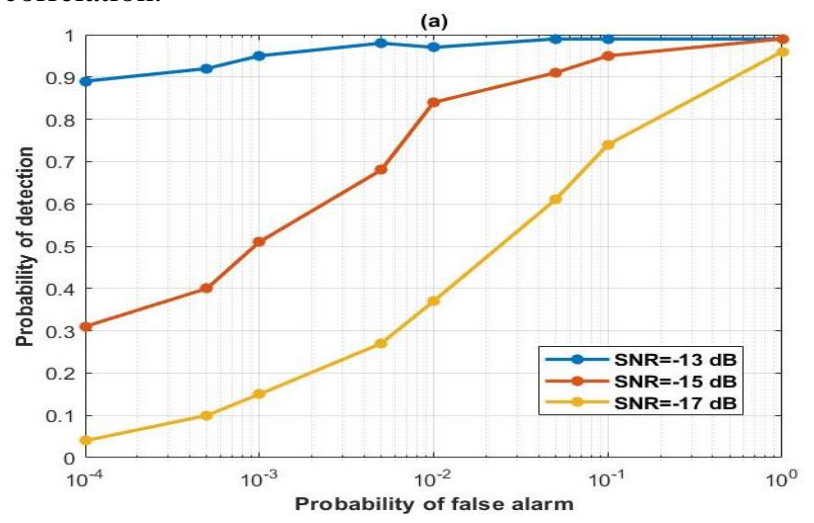


Table 1. A comparison of SNR requirement at $P_{d}=0.9$ to keep $P_{f a}$ a specific value.

\begin{tabular}{l|ccc}
\hline & $\begin{array}{c}\text { single } \\
\text { correlation }\end{array}$ & $\begin{array}{c}\text { double } \\
\text { correlation }\end{array}$ & $\begin{array}{c}\text { triple } \\
\text { correlation }\end{array}$ \\
\hline $\begin{array}{l}\text { SNR (dB) } \\
\text { required for }\end{array}$ & -14.2 & -21.1 & -23.8 \\
Pfa = 0.01 & & & \\
\cline { 1 - 1 } $\begin{array}{l}\text { SNR (dB) } \\
\text { required for }\end{array}$ & & & \\
Pfa =0.05 & -15.1 & -21.7 & -24.2 \\
\cline { 1 - 1 } $\begin{array}{l}\text { SNR (dB) } \\
\text { required for }\end{array}$ & -15.8 & -22.4 & -24.5 \\
Pfa = 0.1 & & & \\
\hline
\end{tabular}
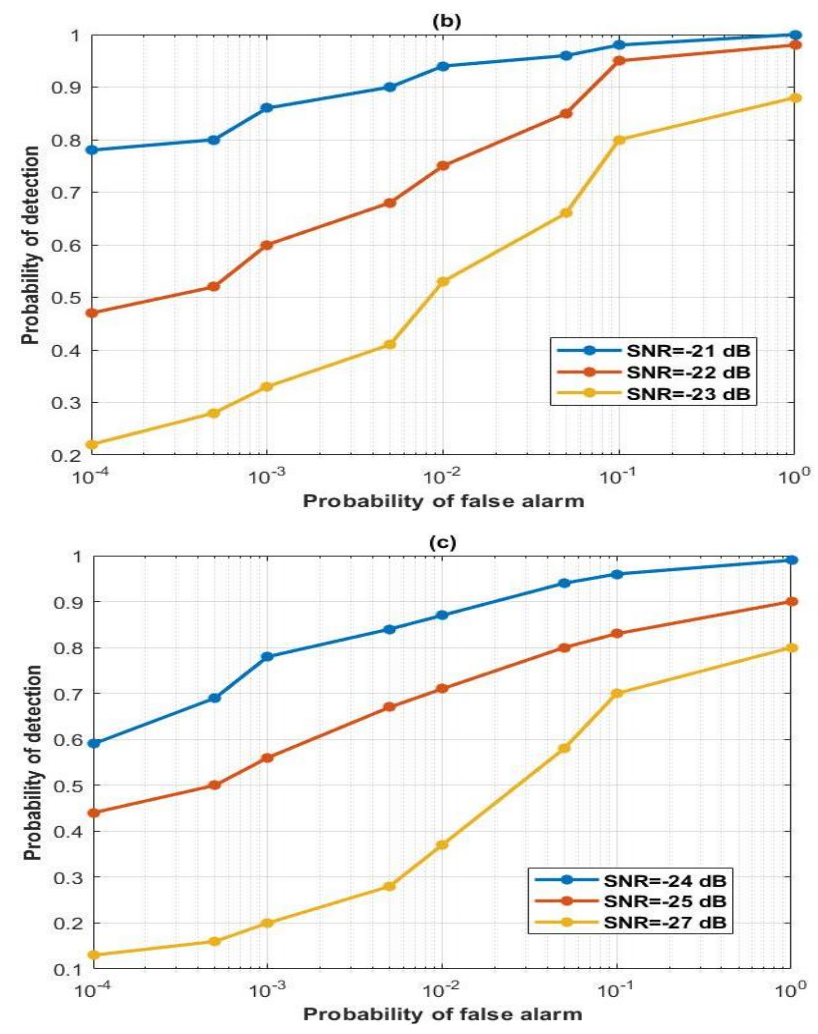

Figure 7. ROC curves of detection using Autocorrelation, (a) single auto-correlation; (b) double auto-correlation; (c) triple auto-correlation.

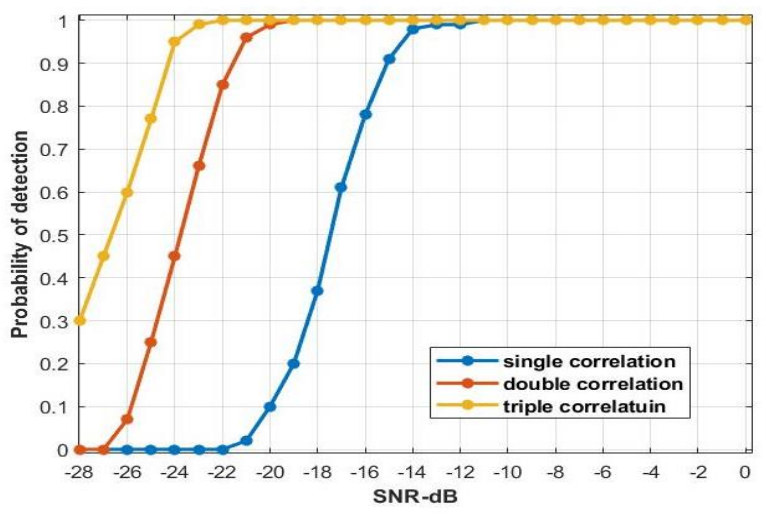

Figure 8. Comparison between multiple auto-correlation function based on probability detection versus SNR for single tone detection.

\section{Conclusions}

This paper investigates the performance of multiple autocorrelation techniques in detecting weak signals. Triple auto-correlation technique showed the best performance over single and double correlation techniques. For $P_{d}=0.9$ and $P_{f a}=0.05$, the double auto-correlation is approximately $6 \mathrm{~dB}$ better than single correlation and triple auto-correction is even better than double correlation by about $3 \mathrm{~dB}$. This gives about $9 \mathrm{~dB}$ improvement of the triple correlation technique compared with the single correlation. Multiple correlation technique, as the single correlation, does not require any pre-information about the signal to be detected. Finally, it was found during simulation that more than triple correlation does not give a significant improvement in the performance of the detector.

\section{Acknowledgements}

The authors wish to thank AL- Al-Mustansiryah University for supporting this research.

\section{Conflict of interest}

The authors declare that this research does not conflict with any other work. 


\section{References}

1. T. Blu and M. Unser. (2003). "Harmonic Spline Series Representation of Scaling Functions". Proceedings of SPIE Applications in Signal and Image Processing, Bellingham: SPIE Press, pp. 120-124.

2. B. Moulin. (2006). "Identification of Nonlinear Aeroelastic and Aeroservoelastic Systems Using High-Order Iterative Technique". 47th AIAA Guidance, Navigation, and Control Conference, Newport: American Institute of Aeronautics and Astronautics Press, pp. 1-16.

3. Wang L, Yang Zh and Waters T.P. (2010). "Structural damage detection using cross correlation functions of vibration response". Journal of Sound and Vibration 329, pp. 5070-5086.

4. Avramchuk V.S and Goncharov V.I. (2013). "Time-frequency Correlation Method for Improving the Accuracy in Detecting Leaks in Pipelines". Advanced Materials Research Vol. 650, Trans Tech Publications, Switzerland. pp. 443-446

5. Jian-fei L, Jia-yu L, Ting-ting Z, Jing-chun L. (2009). "Application of Cross-correlation Algorithm in Radio Weak Signal Detection". Seventh Annual Communication Networks and Services Research Conference.

6. S. M. Kay. (2003). "Fundamentals of Statistical Signal Processing, Volume I: Estimation Theory". Beijing: China Machine Press.

7. T. Moore and B. Sadler. (2006). "Maximum-Likelihood Estimation and Scoring under Parametric Constraints". Army Research Lab, Aldelphi, MD, Tech. Rep. ARL-TR-3805.
8. Yuandong X, et al. (2021). "Autocorrelated Envelopes for early fault detection of rolling bearings". Mechanical Systems and Signal Processing, 146, 106990.

9. Duona Z, et al. (2021). "Learning modulation filter networks for weak signal detection in noise". Pattern Recognition 109, 107590.

10. Lin Lei. (2003). "Micro-computer Automa-tion Detection and System Design [M]". Beijing: Electronics Industry Press. pp. 238-268.

11. Salahdine, F. (2018). "Compressive Spectru-m Sensing for Cognitive Radio Networks". arXiv preprint arXiv: 1802.03674.

12. S. Haykin. (2005) "Cognitive radio: Brainempowered wireless communications". IEEE Journal of Selected Areas in Communicat-ions, vol. 23 , no. 2, pp. 201220.

13. Naila A. (2018). "Application of Correlation Analysis in Weak Signal Detection". IFAC Papers OnLine 51-30. pp. 473-476. 